\title{
Fluoride varnish applications and caries incidence in pre-schoolers
}

\author{
Abstracted from \\ Oliveira BH, Salazar M, Carvalho DM, Falcão A, Campos K, Nadanovsky P. \\ Biannual fluoride varnish applications and caries incidence in preschoolers: \\ a 24-month follow-up randomized placebo-controlled clinical trial. Caries Res 2014; 48: 228-236. \\ Address for correspondence: Branca Heloisa Oliveira, Blv. 28 de Setembro 157, \\ Vila Isabel, Rio de Janeiro, RJ - 20551-030 (Brazil). E-mail: branca.oliveira@gmail.com
}

\section{Question: Does application of fluoride varnishes in preschool children at six month intervals decrease the incidence of caries and produce any adverse effects?}

Design A two year placebo-controlled, double-blind, randomised control trial in 1-4-year-old children.

Intervention Children were recruited from low income families in Rio de Janeiro, Brazil, with access to a fluoridated public water supply. Both groups received oral hygiene instruction along with provision of toothbrushes and 1450ppm F- toothpaste. The control group had a placebo fluoride varnish applied every six months. The intervention group had 22,600ppm F-varnish applied every six months. Clinical examinations for the presence of caries were conducted at baseline and at each subsequent six monthly visit.

Outcome measure The primary outcome was the proportion of children in each group that went on to clinically develop any new carious surfaces using the ICDAS criteria. The number of new carious surfaces was assessed as a secondary outcome, both at the enamel and dentine level. The study also reported on the safety and acceptability of the varnish.

Results Two hundred children participated in the trial, $91 \%$ were followed up at two years and adherence to the six-monthly applications was $84 \%$ in the intervention and $85 \%$ in control groups. The results did not show a significant difference for the development of new lesions between the two groups. In the fluoride varnish group 36\% had developed new dentinal lesions compared to $47 \%$ in the placebo group, a difference of $-10.8 \%(95 \% \mathrm{Cl}-24.9$ to $3.3, \mathrm{p}=0.14)$. In relation to caries increment, at the enamel level, this was a mean of 2.0 teeth in the fluoride varnish group and 2.8 teeth in the placebo group, a difference of $-0.8(95 \% \mathrm{Cl}-2.0$ to $0.4, p=0.19)$. At the dentine level, caries increment was a mean of 1.8 teeth in the fluoride varnish group and 2.5 in the placebo group, a difference of $-0.7(95 \% \mathrm{Cl}-1.9$ to 0.4 , $\mathrm{p}=0.23$ ). Two minor complaints related to taste and appearance of the varnish were recorded and no safety events were reported.

Conclusions Whilst no significant difference was found, the authors note that this does not indicate ineffectiveness of fluoride varnish. Re-assessment of the power calculation following study completion demonstrated that a significantly larger sample size would have been indicated, preventing the drawing of any direct conclusions from the results of this study. The use of fluoride varnish amongst this group appears to be safe and overall well tolerated.

\section{Commentary}

Professionally applied fluoride varnish $(22,600 \mathrm{ppm}$ F-) is a common caries preventive measure. Its use is widely recommended; EAPD, AAPD, SIGN, SDCEP and DoH Oral Health Guidance. ${ }^{1-5}$ In Scotland, its routine use in pre-school children forms a cornerstone of the Scottish Government's Childsmile Programme. ${ }^{6}$

In their trial, Oliveira and colleagues investigated the use of sixmonthly fluoride varnish application amongst high caries risk 1-4 year olds living in Rio de Janeiro, Brazil, (fluoridated public water supply) over a two year period. At enrolment the child's carer attended an oral health educational session, and throughout the trial children had regular toothbrushing instruction (including the free provision of toothbrushes and $1450 \mathrm{ppm}$ F- toothpaste). Following random allocation children received either 22,600ppm Ffluoride varnish or a placebo (identical apart from fluoride content) at six-montly intervals.

Though otherwise methodologically sound, this clearly reported study had a significant issue with its power calculation and sample size. It appears that the study was underpowered, being based on the local caries incidence and an 18\% expected difference between the control and fluoride varnish groups, which gave an indicative sample size of 85 participants per group. A post-study power calculation, based on the actual caries incidence within the study population, indicated that a sample size of 332 in each group was required to detect an effect of the fluoride varnish. This makes any conclusions somewhat uncertain; the lack of statistical difference may have been a result of inadequate numbers of participants to detect a difference and should not be interpreted as a lack of effectiveness. This highlights the importance and difficulty of undertaking an appropriate power calculation at the outset of a study.

Cochrane reviews on the topic of fluoride indicate that the Preventive Fraction (PF) for fluoride varnish versus placebo/no treatment to be $43 \% ;^{7}$ the PF for fluoride varnish in combination with fluoride toothpaste is reported as an additional $10 \%$ over fluoride toothpaste alone. ${ }^{8}$ Based on the figures, a larger sample size was likely required to detect a benefit from fluoride varnish when used in combination with the additional fluorides the participants were exposed to during the trial. Given the trial's low sample size from the initial power calculation, there is a significant risk of a type II error - failure to reject the null hypothesis and not detect an effect when one exists.

The authors question which surfaces benefit most from the anticaries effect of fluoride varnish, querying whether it is sound enamel 
surfaces that derive the most benefit. Their rationale for this is the suggestion from the data that children caries free as baseline showed a greater propensity to remain so at two year follow-up. However, it seems reasonable to suggest that caries free children represent a lower risk group and so a high proportion would be expected to remain caries free at two years.

Whilst the authors obviously took great lengths to conduct a truly placebo-controlled study of fluoride varnish, they included a number of additional preventive interventions (ie oral health education, free toothbrushes, free $1450 \mathrm{ppm}$ F- toothpaste and toothbrushing instruction). It is not unreasonable to include these interventions, particularly if they form part of the routine care received by Brazilian children, and to the trial's credit, they were applied to both groups equally. However, these additional preventive interventions, particularly $1450 \mathrm{ppm}$ F- toothpaste in conjunction with a fluoridated public water supply, are likely to have reduced the estimate of the preventive effect of six-monthly fluoride varnish applications. There was a tendency towards a reduction in the incidence of caries and early enamel lesions in the fluoride varnish arm of the trial. A higher background dmft, lower background fluoride, longer follow-up or an increased sample size may have detected a benefit. This paper highlights why care should always be taken before drawing direct clinical conclusions from an individual study. Fluoride varnishes remain an integral preventive agent when there are no additional sources of fluoride, and may still be of benefit when used in combination with other fluoride interventions.

Alexander J Keightleya , Greig D Taylorb

a Dundee Dental School, University of Dundee, Dundee, Scotland, UK ${ }^{b}$ NHS Ayrshire and Arran, Department of Oral \& Maxillofacial Surgery, University Crosshouse Hospital, Kilmarnock, Scotland, UK

1 American Academy of Paediatric Dentistry. Policy on Use of Fluoride. 2014. http://www.aapd.org (accessed 6 Oct 2014)

2 Scottish Dental Clinical Effectiveness Programme. Prevention and Management of Dental Caries in Children. 2010. http://www.sdcep.org.uk (accessed 6 Oct 2014)

3 Scottish Intercollegiate Guidelines Network (SIGN). Dental interventions to prevent caries in children. Edinburgh, UK, 2014 http://www.sign.ac.uk.

4 European Academy of Paediatric Dentistry. Guidelines on the use of fluoride in children: an EAPD policy document. Eur Arch Paediatr Dent 2009; 10: 129-135.

5 Public Health England. Delivery better oral health: an evidence-based toolkit for prevention. Department of Health: London, UK, 2014.

6 NHS Health Scotland. Childsmile. http://www.child-smile.org.uk (accessed 6 Oct 2014).

7 Marinho VCC, Worthington HV, Walsh T, Clarkson JE. Fluoride varnishes for preventing dental caries in children and adolescents. Cochrane Database Syst Rev 2013; 7: CD002279.

8 Marinho VCC, Higgins JPT, Sheiham A, Logan S. Combinations of topical fluoride (toothpastes, mouthrinses, gels, varnishes) versus single topical fluoride for preventing dental caries in children and adolescents. Cochrane Database Syst Rev 2004; 1: CD002781.

Evidence-Based Dentistry (2014) 15, 83-84. doi:10.1038/sj.ebd.6401045 